\title{
BERGMAN COMPLETENESS OF HYPERCONVEX MANIFOLDS
}

\author{
BO-YONG CHEN*
}

\begin{abstract}
We proved that any hyperconvex manifold has a complete Bergman metric.
\end{abstract}

\section{$\S 1$. Introduction}

Let $M$ be an $n$-dimensional complex manifold. Let $\mathcal{H}$ denote the Hilbert space of holomorphic $n$-forms on $M$ such that $\left|\int_{M} f \wedge \bar{f}\right|<\infty$. Let $h_{0}, h_{1}, \cdots$ be a complete orthonormal basis for $\mathcal{H}$. Then the $2 n-$ form defined on $M \times M$ given by $K_{M}=\sum_{j=0}^{\infty} h_{j} \wedge \bar{h}_{j}$ is called the Bergman kernel form of $M$. Let $z=\left(z_{1}, \cdots, z_{n}\right)$ be a local coordinate system in $M$ and let $K_{M}(z)=K_{M}^{*}(z) d z_{1} \wedge \cdots \wedge d z_{n} \wedge d \bar{z}_{1} \wedge \cdots \wedge d \bar{z}_{n}$ where $K_{M}^{*}$ is a locally defined function. Then $\beta:=\partial \bar{\partial} \log K_{M}^{*}$ is a well-defined Hermitian form of bidegree $(1,1)$, whenever $K_{M}^{*}$ is nonzero. We call $\beta$ the Bergman metric if it is everywhere positive definite. Let us recall

Definition. A complex manifold $M$ is called hyperconvex if there exists a strictly plurisubharmonic (psh) function $\rho: M \rightarrow[-1,0)$ such that $\{x \in M: \rho(x)<c\}$ is relatively compact in $M$ for every $c<0$.

The purpose of this note is to show the following

TheOREM 1. Every hyperconvex manifold has a complete Bergman metric.

Theorem 1 was conjectured by S. Kobayashi [11]. In the special case of bounded hyperconvex domains $\Omega \subset \mathbf{C}^{n}$, it suffices to show that the volume of $\left\{g_{\Omega}(\cdot, y)<-1\right\}$ tends to zero as $y \rightarrow \partial \Omega$, where $g_{\Omega}(\cdot, y)$ denotes the

Received July 16, 2003.

2000 Mathematics Subject Classification: 32F45.

${ }^{*}$ Supported by JSPS. 
pluricomplex Green function of $\Omega$ (cf. Chen [3] and Herbort [8] independently), and this property was verified by Blocki-Pflug [2] (independently Herbort [8]). The case of hyperconvex Riemann surfaces was shown in [4].

Combining with a theorem of Ohsawa-Sibony [13], we obtain

Corollary 2. Every bounded pseudoconvex domain with $C^{2}$ boundary in a complex manifold with positive holomorphic bisectional curvature $\left(\right.$ eg. $\left.\mathbf{P}^{n}\right)$ is Bergman complete.

Greene-Wu [7] proved the the existence of a bounded smooth strictly psh exhaustion function under the following curvature condition. Hence

Corollary 3. Let $M$ be a complete Kähler manifold with a pole o such that its sectional curvature $\mathrm{K}$ is non-positive and in addition satisfies

$$
\mathrm{K} \leq-\frac{1+\epsilon}{r^{2} \log r}
$$

for some constant $\epsilon>0$ outside a compact subset of $M$, where $r$ denotes the distance function based at o. Then $M$ has a complete Bergman metric.

In [7], Bergman completeness has been shown in the case when $M$ is a simply-connected complete Kähler manifold such that the sectional curvature is suitably negatively pinched, for instance, pinched between negative constants. Their result was extended in [4] by only assuming that the curvature is bounded from above by $-A / r^{2}$.

This work was done during the author's stay at Nagoya University. He would like to thank the Department of Mathematics for their hospitality, especially to Professor Takeo Ohsawa for his constant encouragement and discussion. The author also thanks the referee for valuable suggestions.

\section{$\S 2$. Proof of Theorem 1}

Let $g_{M}(\cdot, y)$ be the pluricomplex Green function on $M$, i.e.,

$$
g_{M}(x, y)=\sup \{u(x)\}
$$

where the superum is taken over all negative functions $u \in P S H(M)$ satisfying the property that the function $u-\log |z|$ is bounded from above in a deleted neighborhood of $y$ for some holomorphic local coordinates $z$ centered at $y$, that is, $z(y)=0$. Since $M$ is hyperconvex, $g_{M}(\cdot, y)$ is non-trivial (cf. [4]). Set $d=\partial+\bar{\partial}, d^{c}=i(\bar{\partial}-\partial)$. It is easy to see that $d d^{c}=2 i \partial \bar{\partial}$. As in [2], the following inequality of Blocki is again crucial. 
Proposition 4. (cf.[1]) Let $\Omega$ be a smooth bounded domain in a complex manifold $M$. Assume that $u, v \in C^{\infty}(\bar{\Omega})$ are non-positive psh functions such that $u=0$ on $\partial \Omega$. Then

$$
\int_{\Omega}|u|^{n}\left(d d^{c} v\right)^{n} \leq n !\|v\|_{\infty}^{n-1} \int_{\Omega}|v|\left(d d^{c} u\right)^{n} .
$$

Proof. Note that

$$
\begin{aligned}
\int_{\Omega}(-u)^{n}\left(d d^{c} v\right)^{n}= & n \int_{\Omega}(-u)^{n-1} d u \wedge d^{c} v \wedge\left(d d^{c} v\right)^{n-1} \\
= & n \int_{\Omega}(-u)^{n-1} d v \wedge d^{c} u \wedge\left(d d^{c} v\right)^{n-1} \\
= & n \int_{\Omega}(-u)^{n-1}(-v) d d^{c} u \wedge\left(d d^{c} v\right)^{n-1} \\
& +n(n-1) \int_{\Omega}(-u)^{n-2} v d u \wedge d^{c} u \wedge\left(d d^{c} v\right)^{n-1} \\
\leq & n\|v\|_{\infty} \int_{\Omega}(-u)^{n-1} d d^{c} u \wedge\left(d d^{c} v\right)^{n-1}
\end{aligned}
$$

where the first and third equalities follow from Stokes' theorem and the second one from the fact that the $(1,1)$ parts of $d u \wedge d^{c} v$ and $d v \wedge d^{c} u$ coincide, the inequality follows from $d u \wedge d^{c} u=2 i \partial u \wedge \bar{\partial} u \geq 0$. The desired inequality is obtained by repeating the argument $n-1$ times.

Lemma 5. Let $M$ be a hyperconvex manifold. For any $y \in M$, the following inequality holds:

$$
\int_{M}\left|g_{M}(\cdot, y)\right|^{n}\left(d d^{c} \rho\right)^{n} \leq n !(2 \pi)^{n}|\rho(y)|
$$

Proof. For any positive integer $j$, we set $M_{j}=\{x \in M: \rho(x)<1 / j\}$. Let $y \in M$ and let $g_{M_{j}}(\cdot, y)$ denote the pluricomplex Green function on $M_{j}$ for all sufficiently large $j$. Since $M_{j}$ is hyperconvex, for any fixed $j$, the function $\max \left\{g_{M_{j}}(\cdot, y),-k\right\}+\frac{1}{k}(\rho-1 / j)$ is a continuous strictly psh function and approaches to zero at $\partial M_{j}$ for each integer $k>0$ (cf. [6]). According to a well-known theorem of Richberg [14], there is a smooth psh function $\left\{g_{j, k}\right\}$ on $M_{j}$ such that

$$
\left|g_{j, k}-\max \left\{g_{M_{j}}(\cdot, y),-k\right\}-\frac{1}{k}(\rho-1 / j)\right|<\frac{1}{2 k}|\rho-1 / j|,
$$


which implies $g_{j, k}<0, g_{j, k}(x) \rightarrow 0$ as $x \rightarrow \partial M_{j}$ and $g_{j, k} \rightarrow g_{M_{j}}(\cdot, y)$ as $k \rightarrow \infty$. Hence we can take a sequence of positive numbers $\left\{\lambda_{k}\right\}$ with $\lambda_{k} \rightarrow 0$ as $k \rightarrow \infty$ such that

$$
M_{j-1} \subset \subset M_{j, k}:=\left\{x \in M_{j}: g_{j, k}(x)<-\lambda_{k}\right\} \subset \subset M_{j}
$$

and $M_{j, k}$ has a smooth boundary by Sard's theorem. By Proposition 4, we have

$$
\begin{aligned}
\int_{M_{j-1}}\left|g_{j, k}+\lambda_{k}\right|^{n}\left(d d^{c} \rho\right)^{n} & \leq \int_{M_{j, k}}\left|g_{j, k}+\lambda_{k}\right|^{n}\left(d d^{c} \rho\right)^{n} \\
& \leq n ! \int_{M_{j, k}}|\rho|\left(d d^{c} g_{j, k}\right)^{n} \\
& \leq n ! \int_{M_{j}}|\rho|\left(d d^{c} g_{j, k}\right)^{n} .
\end{aligned}
$$

According to [6], letting $k \rightarrow \infty$, we obtain

$$
\begin{aligned}
\int_{M_{j-1}}\left|g_{M_{j}}(\cdot, y)\right|^{n}\left(d d^{c} \rho\right)^{n} & \leq n ! \int_{M_{j}}|\rho|\left(d d^{c} g_{M_{j}}(\cdot, y)\right)^{n} \\
& =n !(2 \pi)^{n}|\rho(y)|
\end{aligned}
$$

where the equality follows from $\left(d d^{c} g_{M_{j}}(\cdot, y)\right)^{n}=(2 \pi)^{n} \delta_{y}$ on $M_{j}$. The desired inequality is then obtained by letting $j \rightarrow \infty$ since $g_{M_{j}}(\cdot, y) \backslash$ $g_{M}(\cdot, y)$.

Proof of Theorem 1. The existence of the Bergman metric of a hyperconvex manifold was shown in [4]. Take a smooth function $\chi$ on $\mathbf{R}$ such that $\chi=1$ on $(-\infty,-1]$ and $\chi=0$ on $[0, \infty)$. Let $f \in \mathcal{H}$ and $\left\{y_{k}\right\}_{k=1}^{\infty}$ be a sequence of points which has no adherent point in $M$. Set

$$
\begin{aligned}
\eta_{k} & =\chi\left(-\log \left(-g_{M}\left(\cdot, y_{k}\right)+1\right)+\log 2\right) f . \\
\varphi_{k} & =2 n g_{M}\left(\cdot, y_{k}\right)-\log \left(-g_{M}\left(\cdot, y_{k}\right)+1\right)
\end{aligned}
$$

Let us first proceed the proof under the assumption that $\eta_{k}, \varphi_{k}$ are smooth and $\varphi_{k}$ is strictly psh. By the well-known $L^{2}$ estimates (cf. [5], [12]), we 
can solve the equation $\bar{\partial} u_{k}=\bar{\partial} \eta_{k}$ in such a way that

$$
\begin{aligned}
\left|\int_{M} u_{k} \wedge \bar{u}_{k} e^{-\varphi_{k}}\right| & \leq \int_{M}\left|\bar{\partial} \eta_{k}\right|_{\partial \bar{\partial} \varphi_{k}}^{2} e^{-\varphi_{k}} d V_{\varphi_{k}} \\
& \leq C_{1}\left|\int_{A_{k}} f \wedge \bar{f}\right|
\end{aligned}
$$

since

$$
\partial \bar{\partial} \varphi_{k} \geq \frac{\partial g_{M}\left(\cdot, y_{k}\right) \bar{\partial} g_{M}\left(\cdot, y_{k}\right)}{\left(-g_{M}\left(\cdot, y_{k}\right)+1\right)^{2}}
$$

Here $C_{1}$ is a constant depending only on $\sup \left|\chi^{\prime}\right|$ and $A_{k}=\{x \in M$ : $\left.g_{M}\left(\cdot, y_{k}\right)<-1\right\}$. The general case follows from a standard limiting procedure as follows: By a similar argument as in the proof of Lemma 5, one can approximate $g_{M}\left(\cdot, y_{k}\right)$ by a sequence of negative smooth strictly psh functions on $M$ and solve the $\bar{\partial}$-equation with $g_{M}\left(\cdot, y_{k}\right)$ replaced by such functions, then take a limit.

Hence the function $F_{k}=\eta_{k}-u_{k}$ is holomorphic on $M$ which satisfies $F_{k}\left(y_{k}\right)=f\left(y_{k}\right)$ and $\left|\int_{M} F_{k} \wedge \bar{F}_{k}\right| \leq C_{2}\left|\int_{A_{k}} f \wedge \bar{f}\right|$. It follows that

$$
\frac{f\left(y_{k}\right) \wedge \bar{f}\left(y_{k}\right)}{K_{M}\left(y_{k}\right)} \leq C_{2}\left|\int_{A_{k}} f \wedge \bar{f}\right|
$$

For any $\epsilon>0$, there is a relative compact subset $M_{\epsilon}$ so that

$$
\left|\int_{M \backslash M_{\epsilon}} f \wedge \bar{f}\right|<\epsilon .
$$

By Lemma 5, we have

$$
\begin{aligned}
\int_{M_{\epsilon} \cap A_{k}}\left(d d^{c} \rho\right)^{n} & \leq \int_{M}\left|g_{M}\left(\cdot, y_{k}\right)\right|^{n}\left(d d^{c} \rho\right)^{n} \\
& \leq n !(2 \pi)^{n}\left|\rho\left(y_{k}\right)\right| .
\end{aligned}
$$

This shows that one can choose a $k_{\epsilon}$ such that for all $k>k_{\epsilon}$,

$$
\left|\int_{M_{\epsilon} \cap A_{k}} f \wedge \bar{f}\right| \leq \sup _{M_{\epsilon}}\left|\frac{f \wedge \bar{f}}{\left(d d^{c} \rho\right)^{n}}\right| \cdot \int_{M_{\epsilon} \cap A_{k}}\left(d d^{c} \rho\right)^{n}<\epsilon
$$

since $\rho\left(y_{k}\right) \rightarrow 0$ as $k \rightarrow \infty$. By (1)-(3) and the well-known Kobayashi's criterion [10], the Bergman metric on $M$ is complete. 


\section{REFERENCES}

[1] Z. Blocki, Estimates for the complex Monge-Ampere operator, Bull. Pol. Acad. Sci., 41 (1993), 151-157.

[2] Z. Blocki and P. Pflug, Hyperconvexity and Bergman completeness, Nagoya Math. J., 151 (1998), 221-225.

[3] B. Y. Chen, Completeness of the Bergman metric on non-smooth pseudoconvex domains, Ann. Pol. Math., LXXI (1999), 241-251.

[4] B. Y. Chen and J. H. Zhang, The Bergman metric on a Stein manifold with a bounded plurisubharmonic function, Trans. Amer. Math. Soc., 354 (2002), 2997-3009.

[5] J. P. Demailly, Estimations $L^{2}$ pour l'opérateur d'un fibré vectoriel holomorphe semipositiv au dessus d'une variété kählérienne complète, Ann. Sci. Ec. Norm. Sup., 15 (1982), 457-511.

[6] - - Mesures de Monge-Ampère et mesures pluriharmoniques, Math. Z., 194 (1987), 519-564.

[7] R. E. Greene and H. Wu, Function theory on manifolds which possess a pole, Lecture Notes in Mathematics, 699, Springer-Verlag 1979.

[8] G. Herbort, The Bergman metric on hyperconvex domains, Math. Z., 232 (1999), 183-196.

[9] M. Jarnicki and P. Pflug, Bergman completeness of complete circular domains, Ann. Pol. Math., 50 (1989), 219-222.

[10] S. Kobayashi, Geometry of bounded domains, Trans. Amer. Math. Soc. 92 (1959),, 267-290.

[11] -, Hyperbolic Complex spaces, A Series of Comprehensive Studies in Mathematics, 318, Springer-Verlag 1998.

[12] T. Ohsawa, Boundary behavior of the Bergman kernel function on pseudoconvex domains, Publ. RIMS, Kyoto Univ., 20 (1984), 897-902.

[13] T. Ohsawa and N. Sibony, Bounded p.s.h. functions and pseudoconvexity in Kähler manifolds, Nagoya Math. J., 149 (1998), 1-8.

[14] R. Richberg, Steige streng pseudoconvexe Funktionen, Math. Ann., 175 (1968), 257286.

Department of Mathematics

Tongji University

Shanghai 200092

P. R. China

chenbo-yong@lycos.com

CURRENT ADDRESS:

Graduate School of Mathematics

Nagoya University

Chikusa-ku, Nagoya 464-8602

Japan

by-chen@math.nagoya-u.ac.jp 